PHYSICAL REVIEW D 95, 069903(E) (2017)

\title{
Erratum: Effect of relativistic acceleration on localized two-mode Gaussian quantum states
}

[Phys. Rev. D 93, 124031 (2016)]

Mehdi Ahmadi, Krzysztof Lorek, Agata Chęcińska, Alexander R. H. Smith, Robert B. Mann, and Andrzej Dragan (Received 1 March 2017; published 17 March 2017)

DOI: $10.1103 /$ PhysRevD.95.069903

We have found a sign error in our paper. This error affects parts of Sec. III.B and Sec. VI and Eq. (32). Here we present the corrected results. Equations (17), (20), (23), (32), and (40) are modified as

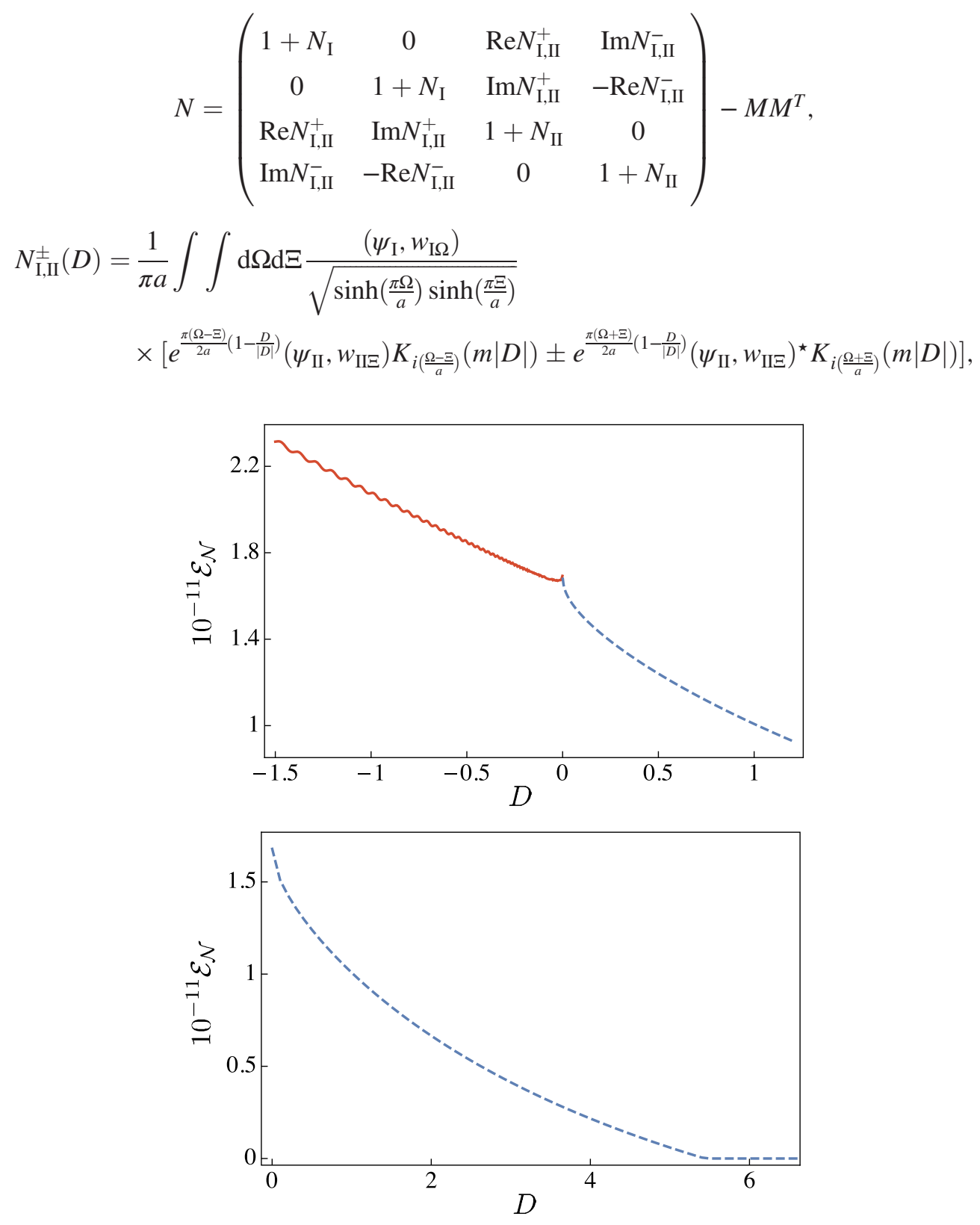

FIG. 1. Logarithmic negativity of the Minkowski vacuum for two counter-accelerated modes, for fixed and equal proper accelerations $\mathcal{A}_{\mathrm{I}}=\mathcal{A}_{\mathrm{II}}=0.1$ as a function of the distance $D$. We have chosen $L=2, m=0.1$, and $\Omega_{0} \approx 5$. In the upper plot, we focus on small wedge separations $D \approx 0$, and the lower plot shows the behavior of the negativity in a larger scale $D>0$. Solid lines correspond to $D<0$ and the dashed lines correspond to $D>0$. 

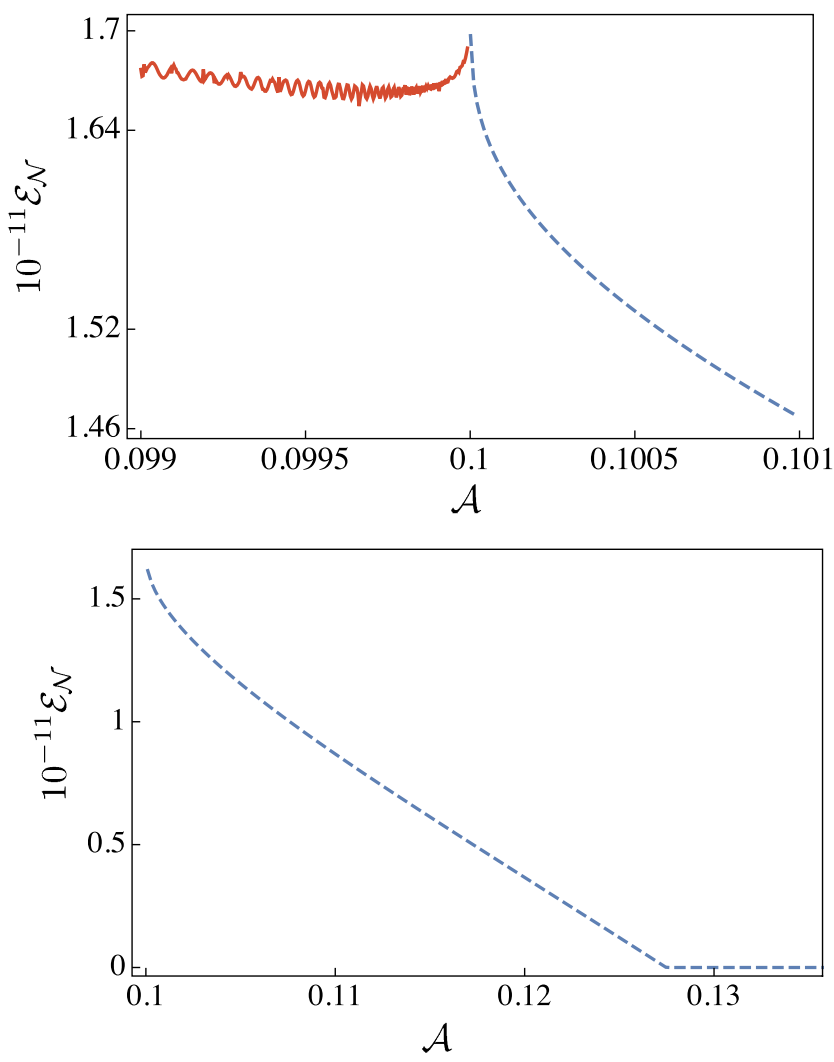

FIG. 2. Logarithmic negativity of the Minkowski vacuum for two counter-accelerated modes, as a function of proper acceleration $\mathcal{A}_{\mathrm{I}}=\mathcal{A}_{\mathrm{II}} \equiv \mathcal{A}$ for $D=20-\frac{2}{\mathcal{A}}$ such that the separation between modes is fixed and equal to 20 . We have chosen $L=2, m=0.1$, and $\Omega_{0} \approx 5$. In the upper plot, we focus on small wedge separations $D \approx 0$ and the lower plot shows the behavior of the negativity in a larger scale, when $D>0$. Solid lines correspond to $D<0$ and the dashed lines correspond to $D>0$.

$$
\begin{aligned}
& N_{\mathrm{I}, \mathrm{II}}^{(( \pm}(D)=\frac{1}{\pi a} \iint \mathrm{d} \Omega \mathrm{d} \Xi \frac{\left(\psi_{\mathrm{I}}, w_{\mathrm{I} \Omega}\right)}{\sqrt{\sinh \left(\frac{\pi \Omega}{a}\right) \sinh \left(\frac{\pi \Xi}{a}\right)}} \\
& \times\left[e^{\frac{\pi}{2 a}\left[(\Omega-\Xi)-(\Omega+\Xi) \frac{D}{|D|}\right]}\left(\psi_{\mathrm{II}}, w_{\mathrm{II} \Xi}\right) K_{i\left(\frac{\Omega+\Xi}{a}\right)}(m|D|) \pm e^{\frac{\pi}{2 a}\left[(\Omega+\Xi)-(\Omega-\Xi) \frac{D}{|D|}\right]}\left(\psi_{\mathrm{II}}, w_{\mathrm{II} \Xi}\right)^{\star} K_{i\left(\frac{\Omega-\Xi}{a}\right)}(m|D|)\right], \\
& N_{\mathrm{I}, \mathrm{II}}^{ \pm}(D)=\frac{1}{\pi} \iint \frac{\mathrm{d} \Omega}{a} \frac{\mathrm{d} \Xi}{a} \frac{F_{\mathrm{I}}\left(\frac{\Omega}{a}, \mathcal{A}_{\mathrm{I}}\right)}{\sqrt{\sinh \left(\frac{\pi \Omega}{a}\right) \sinh \left(\frac{\pi \Xi}{a}\right)}} \\
& \times\left[e^{\frac{\pi(\Omega-\Xi)}{2 a}\left(1-\frac{D}{|D|}\right)} F_{\mathrm{II}}\left(\frac{\Xi}{a}, \mathcal{A}_{\mathrm{II}}\right) K_{i\left(\frac{\Omega-\Xi}{a}\right)}(m|D|) \pm e^{\frac{\pi(\Omega+\Xi)}{2 a}\left(1-\frac{D}{|D|}\right)} F_{\mathrm{II}}^{\star}\left(\frac{\Xi}{a}, \mathcal{A}_{\mathrm{II}}\right) K_{i\left(\frac{\Omega+\Xi}{a}\right)}(m|D|)\right], \\
& \sigma^{(d)}=\left(\begin{array}{cccc}
1+N_{\mathrm{I}} & 0 & \operatorname{Re} N_{\mathrm{I}, \mathrm{II}}^{+} & \operatorname{Im} N_{\mathrm{I}, \mathrm{II}}^{-} \\
0 & 1+N_{\mathrm{I}} & \operatorname{Im} N_{\mathrm{I}, \mathrm{II}}^{+} & -\operatorname{Re} N_{\mathrm{I}, \mathrm{II}}^{-} \\
\operatorname{Re} N_{\mathrm{I}, \mathrm{II}}^{+} & \operatorname{Im} N_{\mathrm{I}, \mathrm{II}}^{+} & 1+N_{\mathrm{II}} & 0 \\
\operatorname{Im} N_{\mathrm{I}, \mathrm{II}}^{-} & -\operatorname{Re} N_{\mathrm{I}, \mathrm{II}}^{-} & 0 & 1+N_{\mathrm{II}}
\end{array}\right) .
\end{aligned}
$$

In Eqs. (21) and (22), $N_{\mathrm{I}, \mathrm{II}}(D)$ changes to $N_{\mathrm{I}, \mathrm{II}}^{ \pm}(D)$ and in Eqs. (24) and (25), $N_{\mathrm{I}, \mathrm{II}}^{((}(D)$ changes to $N_{\mathrm{I}, \mathrm{II}}^{(( \pm}(D)$. The new definition of $\Delta$ in Eq. (41) is given as 


$$
\Delta \equiv\left(1+N_{\mathrm{I}}\right)^{2}+\left(1+N_{\mathrm{II}}\right)^{2}+\operatorname{Re} N_{\mathrm{I}, \mathrm{II}}^{+} \operatorname{Re} N_{\mathrm{I}, \mathrm{II}}^{-}+\operatorname{Im} N_{\mathrm{I}, \mathrm{II}}^{+} \operatorname{Im} N_{\mathrm{I}, \mathrm{II}}^{-}
$$

After taking into account the corrections above, we have replaced Figs. 7 and 8 of the original paper with Figs. 1 and 2. For the new plots, we have performed the exact numerics rather than the approximations in Eq. (42). Qualitatively, for the case of counter-accelerated modes, the conclusions remain valid, except that we observe the sudden death of entanglement only for $D>0$.

Finally, for the scenario wherein both output modes are accelerated in the same direction, we have numerically evaluated the logarithmic negativity of the output state of the channel for a range of parameters and found zero negativity both for $D=0$ and $D \neq 0$. This strongly suggests that there is no vacuum entanglement within the parallel accelerations setup. 